\title{
Benefícios da intervenção fisioterapêutica no fortalecimento dos músculos do assoalho pélvico, após o parto normal
}

\author{
Benefits of physiotherapy in the strengthening \\ of pelvic muscle, after normal birth
}

Cleudimar da Costa Silva ${ }^{1}$ Virginia Leyla Santos Costa URTIGA ${ }^{\text {II }}$

André Pontes SiLva III

${ }^{1}$ Instituto de Educação Superior Raimundo Sá (URSA), Picos/PI - Brasil

II Universidade Federal do Ceará (UFC), Fortaleza/CE - Brasil

III Universidade Federal do Maranhão, Maranhão/MA - Brasil
RESUmo Este estudo teve como objetivo avaliar os benefícios do tratamento fisioterapêutico no fortalecimento dos músculos do assoalho pélvico, após o parto normal. Trata-se de um estudo de caso do tipo intervencional e comparativo. A população foi composta por quatro pacientes voluntárias com histórico de parto normal residentes no município de Picos, no Estado do Piauí. O tratamento foi realizado no Centro de Atendimento da Faculdade Raimundo de Sá, localizado na Rua Padre Madeira, 201, centro, Picos-Piauí, no período de março e abril de 2017. Os dados foram analisados com base nas informações coletadas por meio de avaliação e reavaliação da palpação bidigital executada pelo(a) mesmo(a) examinador(a), escala de Oxford modificada e pelo visor emitido pelo biofeedback perineal, além da aplicação do questionário de satisfação das pacientes no final do atendimento. A partir dos resultados e discussão desta pesquisa evidenciamos: que a utilização do biofeedback associada ao treinamento dos músculos do assoalho pélvico é eficiente para o fortalecimento dos músculos do assoalho pélvico após o parto normal; aumento da força isométrica dos músculos do assoalho pélvico e controle urinário; e, aumento do prazer sexual das pacientes. Vale ressaltar que, por se tratar apenas de um relato de caso, este artigo não institui um protocolo intervencional para tratamento de possíveis complicações no pós-parto, uma vez que, a amostra utilizada, não representa uma fração significativa da população do Estado do Piauí.

Palavras-chave: Puerpério. Assoalho Pélvico. Incontinência URINÁRIA.

Abstract The objective was to evaluate the benefits of physical therapy in strengthening the pelvic floor muscles after normal delivery. It is a case study of the interventional and comparative type. The population was composed of four volunteer patients with normal birth history, living in the municipality of Picos, State of Piauí. The treatment was carried out at the Raimundo de Sá Faculty Center, located at Rua Padre Madeira, 201, center, Picos-Piauí, in the period of March and April 2017. Data were analyzed based on the information collected through evaluation and reassessment of the bidigital palpation performed by the same examiner, modified Oxford scale and by the display of the perineal biofeedback, in addition to the application of the patient satisfaction questionnaire at the end of the care. From the results and discussion of 
this research we show: that the use of biofeedback associated with the training of the pelvic floor muscles is efficient for the strengthening of pelvic floor muscles after normal delivery; increased isometric strength of the pelvic floor muscles and urinary control; and, increase of the sexual pleasure of the patients. It is worth noting that, because it is only a case report, this article does not establish an intervention protocol for the treatment of possible postpartum complications, since the sample used does not represent a significant fraction of the population of the State of Piauí.

Keywords: Puerperium. Pelvic Floor. Urinary Incontinence.

\section{INTRODUÇÃO}

Segundo a literatura das ciências biológicas e da saúde, a pelve humana constitui a porção mais inferior do core, e ocupa uma posição intermediária entre o centro de gravidade e os membros inferiores; sua formação ocorre a partir dos ossos: sacro e cóccix, e é composta pelos ossos ílio, ísquio e púbis; que se articulam na sínfise púbi$\mathrm{ca}$ anteriormente e ao sacro posteriormente: articulação sacro ilíacas. ${ }^{1,2}$

Como o Assoalho Pélvico (AP) desempenha o papel de sustentar os órgãos internos, contendo as estruturas: útero, bexiga e reto, formada por músculos, fáscias e ligamentos; esse complexo sofre modificações durante a gravidez e parto, para permitir a passagem do feto, manter a continência urinária e fecal. ${ }^{3}$

A gravidez e o parto são acontecimentos sociais que integram a vivência reprodutiva de mulheres, e nesse período, o corpo passa por adaptações fisiológicas profundas, as quais envolvem todos os sistemas. ${ }^{4}$ No percurso da gestação, como se pode imaginar, os MAP sofrem sobrecarga, já que nesse momento, além de sustentar o peso constante dos órgãos pélvicos, os MAP precisam sustentar também o peso do bebê e seus anexos embrionários. ${ }^{5}$
Durante a gestação, há um aumento no tamanho e no peso dos rins e a bexiga é progressivamente elevada pelo útero aumentando nos dois últimos trimestres da gravidez, tornando-se um órgão intra-abdominal; os Músculos do Assoalho Pélvico (MAP) são afetados significativamente, justificando assim a hipotonia dessa cadeia muscular no pós-parto, que pode desencadear o surgimento de Incontinência Urinária (IU) e fecal, disfunções sexuais e prolapsos de órgãos pélvicos. ${ }^{6}$

No momento do parto, para que a cabeça do bebê alcance a superfície pélvica, o AP e outras estruturas como a vagina, a bexiga e a uretra sofrem estiramentos; alongamentos; compressões; e dilatações, formando então o canal de parto, essas alterações podem causar danos à inervação dos músculos pélvicos superficiais durante o parto, aumentando o risco de uma IU e disfunções do AP pós-parto.

Um estudo ${ }^{8}$ evidenciou que a presença de disfunções nos MAP pode causar IU na gestação e no puerpério, suscitando a necessidade de avaliação da capacidade de contração dos MAP, para então, instituir-se um programa de treinamento que busque melhorar a função muscular e prevenir/tratar essas disfunções.

Por sua vez, o puerpério é caracterizado por um período de intensas alterações 
no corpo da mulher durante o pós-parto, as quais podem durar vários meses para atingir o seu restabelecimento., 2

Nos últimos anos, em virtude desse contexto, a fisioterapia uroginecológica tem se destacado no tratamento da hipotonia do Assoalho Pélvico (AP), após o parto, uma vez que esta dispõe recursos efetivos que possibilitam a recuperação da estabilidade no AP após o parto normal. ${ }^{10}$ Sabendo que o parto normal pode comprometer os MAP e desencadear disfunções, quais são os benefícios do fortalecimento dos MAP em mulheres, após o parto normal? Este estudo tem como objetivos avaliar os benefícios do tratamento fisioterapêutico no fortalecimento dos músculos do assoalho pélvico, após o parto normal.

\section{Metodologia}

Trata-se de um estudo de caso do tipo intervencional e comparativo. A população foi composta por quatro pacientes voluntárias com histórico de parto normal residentes no município de Picos, no Estado do Piauí. O tratamento foi realizado no Centro de Atendimento da Faculdade Raimundo de Sá, localizado na Rua Padre Madeira, 201, centro, Picos-Piauí, no período de março e abril de 2017.

Este estudo contém o tratamento ético de uma pesquisa envolvendo seres humanos, uma vez que foi utilizado Termo de Consentimento Livre e Esclarecido (TCLE), que possibilitou o Certificado de Apresentação para Apreciação Ética (CAAE) de número "65095916.0.0000.8057", e, aprovação do Comitê de Ética em Pesquisa (CEP) pelo número do parecer "2389106".
Critérios de inclusão: pacientes com tempo entre quarenta e sessenta dias de pós-parto normal; faixa etária de 18 a 25 anos de idade, que não realizaram tratamento fisioterapêutico nos MAP durante a gestação. O ponto de corte já relacionado se justifica pelo fato de que, quarenta dias após o parto normal, o organismo inicia a "normalização" da função reprodutiva, nesse período, a avaliação dos MAP parece mais eficaz no que se refere a comparações posteriores. ${ }^{4}$

Critérios de exclusão: pacientes que optaram por cesárea; parto prematuro; tabagistas; mulheres com distúrbio psiquiátrico ou comprometimento cognitivo.

\section{Procedimento do estudo}

Inicialmente, as pacientes foram submetidas a uma avaliação fisioterapêutica realizada através de anamnese, que investigou as queixas principais, história da gestação e pós-parto, história social, fluxo urinário e atividade sexual.

Durante a inspeção, observamos nas estruturas externas dos MAP presença ou ausência de contração voluntária ou movimento do períneo durante o comando verbal, presença de cicatrizes ou feridas, distopias genitais, eritemas e alteração na cor da mucosa.

Foi avaliada a ativação muscular do AP por meio da palpação bidigital de um(a) fisioterapeuta. A Escala de Oxford modificada, e, o biofeedback ${ }^{1}$ perineal foram

\footnotetext{
Marca Quark ${ }^{\oplus}$, modelo 996-2, averiguado pelo Instituto de Pesos e Medidas, com graduação de 0 a $46,4 \mathrm{mmHg}$.
} 
utilizadas(o) para aferir o pico de força isométrica, uma vez que, por intermédio desses instrumentos é possível avaliar padrões de atividade muscular.

Após a avaliação fisioterapêutica, foram realizados dez atendimentos com o biofeedback perineal associado com o treinamento dos músculos do assoalho pélvico (TMAP) cones vaginais, na frequência de três vezes por semana, com duração de 30-40 minutos cada atendimento; o biofeedback foi utilizado com os seguintes parâmetros: escala de pressão A, tempo de sustentação quatro segundos, tempo de repouso x 2 e tempo de terapia dez minutos. Logo após foi colocado um cone vaginal e solicitado que a paciente caminhasse mantendo o cone na vagina, para assim verificar possível ganho de propriocepção, coordenação e força isométrica.

Após cinco sessões, fez-se a utilização do biofeedback com os seguintes parâmetros: tempo de sustentação oito segundos, tempo de repouso $\mathrm{x} 2$ e tempo de terapia vinte minutos com a paciente deitada, pés apoitados na maca e uma bola entre as pernas. Foi solicitado que a paciente apertasse a bola e os músculos do AP. Em seguida, foi aplicado um cone vaginal e duas séries de agachamento com dez repetições, para verificar a habilidade em manter o cone na vagina, e, evidenciar o desenvolvimento de propriocepção, coordenação e força isométrica.

Os dados foram analisados com base nas informações coletadas por meio de uma reavaliação da palpação bidigital executada pelo(a) mesmo(a) examinador(a), escala de Oxford modificada e pelo visor emitido pelo biofeedback perineal, além da aplicação do questionário de satisfação das pacientes no final do atendimento.

\section{Resultados E Discussão}

A respeito do perfil das participantes da pesquisa, estas apresentavam idade entre 18 e 25 anos, idade gestacional de 38 a 40 semanas e, apenas uma das pacientes referiu obesidade após a gravidez, sobre o estado civil todas eram casadas e não praticavam exercício físico (Tabela 1).

Em relação à perda urinária relatada pelas pacientes antes e após a intervenção (Tabela 1), três pacientes apresentaram IU, entre elas, duas de esforço e uma de urgência; foi possível notar que a IU tornou-se alvo de inúmeros estudos, haja vista que além de caracterizar uma perda involuntária de urina, gera desconforto social e higiênico.

Uma das funções dos MAPs se refere à manutenção da continência urinária, por conseguinte sua disfunção desencadeia a IU, muito frequente no período gestacional e no puerpério; o déficit de consciência corporal nessa cadeia muscular pode ser uma causa plausível e, por intermédio de exercícios, a fisioterapia atuará na conscientização e aprendizagem motora no que se refere à contração dos MAP. ${ }^{11}$

O parto vaginal tem sido apontado como um dos principais fatores de risco para o surgimento da IU no pós-parto, em razão do risco de lesão nas estruturas de suporte de tecido conectivo da pelve, nas estruturas vasculares, nos nervos e/ou músculos do trato unitário. ${ }^{12}$

O tratamento da IU e de outras disfunções do AP pode ser clínico, cirúrgico ou conservador, todavia, nem sempre as intervenções cirúrgicas são as mais indicadas. ${ }^{7}$

Nesse sentido, a atuação preventiva com exercícios durante toda a gestação e no pós-parto - imediato e tardio -, principalmente para aquelas mulheres que tiveram alguma 
disfunção ainda na gestação, parece a melhor opção. O tratamento fisioterapêutico deverá estabilizar o AP, aumentar sua sensibilidade, fortalecê-lo por meio de exercícios de contração e relaxamento. ${ }^{13}$

Quando questionadas sobre a qualidade da vida sexual (Tabela 1), as voluntárias relataram dor durante a relação sexual após o parto normal, porém, após a intervenção do tratamento o qual este estudo relata, houve melhora significativa na qualidade da vida sexual das pacientes.

Corroborando essa informação, um estudo $^{14}$ analisou duzentas puérperas que retomaram a vida sexual ativa, os resultados certificaram que 76,\% apresentavam disfunções sexuais antes da gravidez, durante e após o parto, respectivamente.

$\mathrm{Na}$ avaliação dos MAP, a palpação transvaginal é considerada um método eficaz na identificação da capacidade de contração. Existem diversas escalas de avaliação da função dos MAP por meio da palpação vaginal, entretanto a mais utilizada é a Escala de Oxford Modificada. ${ }^{8}$

Baseando-se na palpação transvaginal de acordo com a Escala Oxford - modifica$\mathrm{da}$, as quatro voluntárias apresentaram aumento de força isométrica (Tabela 1); por meio de um ensaio clínico randomizado, uma pesquisa ${ }^{15}$ evidenciou que puérperas praticantes de exercício físico para os MAP apresentam evolução significativa quando comparadas às que não praticam.

Durante a inspeção, foi possível observar nas estruturas externas do MAP presença e/ou ausência de contração voluntária ou movimento do períneo para dentro em resposta ao comando verbal. Conforme descreve a Tabela 1, no início da intervenção, ao fazer a contração solicitada, as quatro voluntárias usaram músculos acessó- rios, bem como, relataram dificuldade no controle neuromuscular no que se refere à contração isolada e específica dos músculos do AP. Ao final das sessões, todas conseguiram fazer as contrações corretas.

Quando se fala em disfunções neuromusculares relacionada à consciência corporal, a coordenação motora e debilidades neuromusculares podem ter o seu quadro revertido quando treinadas a desenvolver a habilidade necessária para atingir uma aprendizagem motora complexa. ${ }^{16}$

O biofeedback é capaz de determinar os potenciais de ação das contrações musculares do AP e exibir essas intensidades por meio de uma escala linear de pressão representada por uma escala luminosa de LEDs, a qual acompanha, em tempo real, o comportamento das contrações musculares; é possível controlar o tempo de terapia, o tempo de contração e o tempo de repouso, além de fixar uma pressão-objetivo a ser atingida durante a terapia; o Biofeedback permite a reeducação ativa controlada e exige a cooperação da paciente. ${ }^{17}$

O biofeedback é bastante utilizado na reeducação motora do AP, pois proporciona à paciente uma resposta, por meio de sinais luminosos, numéricos e sonoros, permitindo que a mesma realize autoavaliação do MAP sobre o movimento, que possa ser medido, ensinado e aprendido. ${ }^{18}$

Em seus estudos, Batista et al. $(2011)^{8}$ confirmam a eficiência do biofeedback, a autora constatou um aumento crescente na amplitude eletromiográfica a cada contração realizada no percurso da intervenção da qual se trata.

Quanto à representação do potencial de ação da contração muscular dos MAP, por meio do biofeedback, nossos dados exprimem resultados positivos na esta- 
bilização e fortalecimento do AP após o treinamento de contração e relaxamento realizado neste estudo; todas as pacientes responderam positivamente sobre a pos- sibilidade de indicação da intervenção fisioterapêutica no fortalecimento dos músculos do assoalho pélvico, após o parto normal (Tabela 1).

Tabela 1: $\mathrm{P}=$ Pacientes 1-4. MAP=Músculos do Assoalho Pélvico.

Dados da coleta e resultados desta pesquisa.

\begin{tabular}{lcccc}
\hline Variáveis & Caracterização das pacientes & investigadas. Picos & - PI, 2017. (n=4) & \\
Perfil & $\mathbf{P 1}$ & $\mathbf{P 2}$ & $\mathbf{P 3}$ & $\mathbf{P 4}$ \\
Idade & 19 & 21 & 25 & 18 \\
Idade Gestacional & 39 & 39 & 40 & 38 \\
Obesidade & Não & Não & Não & Sim \\
Estado Civil & Casada & Casada & Casada & Casada \\
Profissão & Estudante & Dona de & Administradora & Comerciante \\
& & Casa & & Não \\
Exercício Físico & Não & Não & Não
\end{tabular}

Perda urinária relatada pelas pacientes. Picos-PI, 2017. ( $n=04)$

$\begin{array}{lcccc}\text { Perda urinária } & \text { P1 } & \text { P2 } & \text { P3 } & \text { P4 } \\ \text { Antes } & \text { Sim } & \text { Sim } & \text { Sim } & \text { Não } \\ \text { Depois } & \text { Não } & \text { Não } & \text { Não } & \text { Não }\end{array}$

Caracterização da vida sexual relatada pelas pacientes. Picos-PI, 2017. (n=04)

$\begin{array}{lcccc}\text { Relação Sexual } & \text { P1 } & \text { P2 } & \text { P3 } & \text { P4 } \\ \text { Antes } & \text { Boa } & \text { Ruim } & \text { Boa } & \text { Boa } \\ \text { Depois } & \text { Ótima } & \text { Boa } & \text { Ótima } & \text { Ótima }\end{array}$

Palpação Bidigital baseada na escala de Oxford - modificada. Picos-PI, 2017. (n=04)

$\begin{array}{lcccc}\text { Contração MAP } & \text { P1 } & \text { P2 } & \text { P3 } & \text { P4 } \\ \text { Antes } & \text { Grau 2 } & \text { Grau 2 } & \text { Grau 2 } & \text { Grau 3 } \\ \text { Depois } & \text { Grau 3 } & \text { Grau 4 } & \text { Grau 4 } & \text { Grau 4 }\end{array}$

Inspeção do controle neuromuscular para a contração dos MAP. Picos-PI, 2017. (n=04)

$\begin{array}{lcccc}\text { Dificuldade } & \text { P1 } & \text { P2 } & \text { P3 } & \text { P4 } \\ \text { Antes } & \text { Sim } & \text { Sim } & \text { Sim } & \text { Sim } \\ \text { Depois } & \text { Não } & \text { Não } & \text { Não } & \text { Não }\end{array}$

Visor do Biofeedback representando o potencial de ação da contração muscular. Picos-PI, 2017.

$\begin{array}{lcccc} & & (\mathrm{n}=04) & & \text { P3 } \\ \text { Potenciais de ação } & \text { P1 } & \text { P2 } & \text { P3 } \\ \text { Antes } & \text { Escala A } & \text { Escala A } & \text { Escala A } & \text { Escala B } \\ & 2 \mathrm{cmH} 2 \mathrm{O} & 4 \mathrm{cmH} 2 \mathrm{O} & 1 \mathrm{cmH} 2 \mathrm{O} & 8 \mathrm{cmH} 2 \mathrm{O} \\ \text { Depois } & \text { Escala A } & \text { Escala A } & \text { Escala A } & \text { Escala B } \\ & 5 \mathrm{cmH} 2 \mathrm{O} & 8 \mathrm{cmH} 2 \mathrm{O} & 6 \mathrm{cmH} 2 \mathrm{O} & 16 \mathrm{cmH} 2 \mathrm{O}\end{array}$

Pergunta aplicada às participantes, após a intervenção Fisioterapêutica. Picos-PI, 2017. (n=04).

\begin{tabular}{lcccc} 
Você indicaria? & P1 & P2 & P3 & P4 \\
Resposta & Sim & Sim & Sim & Sim \\
\hline
\end{tabular}

Fonte: Elaborada pelos responsáveis do estudo, Picos-Piauí, 2017. 


\section{CONSIDERAÇõES FINAIS}

A partir dos resultados e discussão desta pesquisa evidenciamos: que utilização do biofeedback associado ao TMAP é eficiente para o fortalecimento dos MAP, após o parto normal; aumento da força isométrica dos MAP e controle urinário; e, aumento do prazer sexual das pacientes.

Além disso, foi possível verificar também que o fortalecimento dos MAP se faz necessário antes da gestação, durante a gestação e após o parto normal, uma vez que essa cadeia muscular auxilia a sustentação das vísceras e útero e, nesse sentido, o fortalecimento dos MAP pode evitar lesões no AP e facilitar a recuperação no pós-parto.

Sobre as limitações deste estudo, podemos considerar que este limitou-se a uma intervenção em quatro pacientes, uma quantidade relativamente pequena que, por sua vez, não determina um caráter prescritivo de tratamento para os casos apresentados ao longo do trabalho.

Desse modo, estas informações não devem ser consideradas isoladamente, mas como auxiliares para prevenir, tratar, informar e conscientizar fisioterapeutas e pacientes, sobre a importância do fortalecimento dos MAP, após o parto normal.

Ao longo da coleta de dados, avaliações, resultados e discussão deste trabalho foi possível avaliar os benefícios do tratamento fisioterapêutico no fortalecimento dos músculos do assoalho pélvico após parto normal; vale ressaltar que, por se tratar apenas de um relato de caso, este artigo não institui um protocolo intervencional para tratamento de possíveis complicações no pós-parto, uma vez que, a amostra utilizada, não representa uma fração significativa da população do Estado do Piauí.

\section{REFERÊNCIAS}

1. Rett MT, et al. Existe diferença na contratilidade da musculatura do assoalho pélvico feminino em diversas posições. Rev Bras Ginecol Obstet, v. 27, n. 1, p. 20-3, 2005. Disponível em: http:// www.scielo.br/pdf/\%0d/rbgo/v27n1/24287.pdf. Acesso em: 10 set. 2018.

2. Baracho E. Fisioterapia Aplicada à Obstetrícia, Uroginecologia e Aspectos de Mastologia, 5 . ed. Rio de Janeiro: Guanabara Koogan, 2012.

3. Moreno AL. Fisioterapia em Uroginecologia, 2. ed. Barueri, São Paulo: Manole, 2009.

4. Padilha JF. Avaliação da modulação autonômica cardíaca no processo de enchimento da bexiga de mulheres com incontinência urinária: perspectiva da fisioterapia. [Dissertação de Mestrado]. Florianópolis: Universidade do Estado de Santa Catarina - Centro de Ciências da Saúde e do Esporte, 2015. Disponível em: http://tede.udesc.br/bitstream/tede/943/1/_juliana_ padilha_.pdf. Acesso em: 9 set. 2018.

5. Silveira LC, Segre CAM. Exercício físico durante a gestação e sua influência no tipo de parto. Einstein (16794508), v. 10, n. 4, 2012. Disponível em: http://www.scielo.br/pdf/eins/v10n4/ pt_v10n4a03.pdf. Acesso em: 8 set. 2018.

6. Knorst MR, et al. Intervenção fisioterapêutica em mulheres com incontinência urinária associada ao prolapso de órgão pélvico. Rev Bras Fisioter, v. 16, n. 2, p. 102-7, 2012. Disponível em: http://www.scielo.br/pdf/rbfis/v16n2/a04v16n2. Acesso em: 9 set. 2018.

7. Glisoi SFN, Girelli, P. Importância da fisioterapia na conscientização e aprendizagem da contração da musculatura do assoalho pélvico em mulheres com incontinência urinária. Rev Bras 
Clin Med, v. 9, n. 6, p. 408-13, 2011. Disponível em: http://www.sbcm.org.br/revistas/rbcm/ rbcm-2011-06.pdf. Acesso em: 9 set. 2018.

8. Batista RL, et al. Biofeedback na atividade eletromiográfica dos músculos do assoalho pélvico em gestantes. Rev Bras Fisioter, v. 15, n. 5, p. 386-92, 2011. Disponível em: http://www.scielo.br/ pdf/rbfis/2011nahead/pt_aop026-11.pdf. Acesso em: 9 set. 2018.

9. Strapasson MR, Nedel MN. B. Puerpério imediato: desvendando o significado da maternidade. Revista Gaúcha de Enfermagem, v. 31, n. 3, p. 521, 2010. Disponível em: http://www.scielo. br/pdf/rgenf/v31n3/v31n3a16. Acesso em: 8 set. 2018.

10. Moura JFAL, Marsal AS. Cinesioterapia para o fortalecimento do assoalho pélvico no período gestacional. Revista Visão Universitária, v. 3, n. 1, p. 186-201, 2015. Disponível em: http://www. visaouniversitaria.com.br/ojs/index.php/home/article/viewFile/57/56. Acesso em: 9 set. 2018.

11. Guerra TEC, et al. Atuação da Fisioterapia no tratamento de Incontinência Urinária de Esforço. Revista Femina, v. 42, n. 6, p. 63-68, 2014. Disponível em: <http://files.bvs.br/ upload/S/0100-7254/2014/v42n6/a4823.pdf>. Acesso em: 9 set. 2018.

12. Martins AB, Ribeiro J., Soler ZASG. Proposta de exercícios físicos no pós-parto. Um enfoque na atuação do enfermeiro obstetra. Investir. Educ. Enferm, Medellín, v. 29, n. 1, p. 40-46, 2011. Disponível em: http://www.scielo.org.co/pdf/iee/v29n1/v29n1a05.pdf. Acesso em: 9 set. 2018.

13. Felicíssimo MF, et al. Fatores limitadores à reabilitação da musculatura do assoalho pélvico em pacientes com incontinência urinária de esforço. Acta Fisiátrica, v. 14, n. 4, p. 233-236, 2016. Disponível em: http://www.periodicos.usp.br/actafisiatrica/article/download/102869/101160. Acesso em: 10 set. 2018.

14. Holanda JBL, et al. Disfunção sexual e fatores associados relatados no período pós-parto. Acta paul. Enferm., São Paulo, v. 27, n. 6, p. 573-578, dez. 2014. Disponível em: http://www.scielo.br/ pdf/ape/v27n6/en_1982-0194-ape-027-006-0573.pdf. Acesso em: 9 set. 2018.

15. Assis TR, et al. Efeito de um programa de exercícios para o fortalecimento dos músculos do assoalho pélvico de multíparas The effect of an exercise program to strengthen pelvic floor muscles in multiparous women. Revista Brasileira de Ginecologia e Obstetrícia, v. 35, n. 1, p. 10-15, 2013. Disponível em: http://www.scielo.br/pdf/rbgo/v35n1/03.pdf. Acesso em: 9 set. 2018.

16. Silva AP, et al. Os principais aspectos do Transtorno do Desenvolvimento da Coordenação nas Atividades da Vida Diária de uma criança em fase de crescimento: um consenso científico. Batatais: Claretiano, v. 6, n. 1, p. 37-45, 2017. Disponível em: http://www.claretianobt.com.br/revista/fpmzphuzfvwv. Acesso em: 9 set. 2018.

17. Santana LS, et al. Utilização dos recursos fisioterapêuticos no puerpério: revisão da literatura. Femina, v. 39, n. 5, 2011. Disponível em: http://files.bvs.br/upload/S/0100-7254/2011/v39n5/ a2506.pdf. Acesso em: 9 set. 2018.

18. De Liz NA, et al. Fisioterapia no período puerperal: revisão sistemática. Corpvs, v. 1, n. 27, p. 09-20, 2015. Disponível em: http://publica-estaciofic.com.br/revistas/index.php/corpvs/article/ view/65. Acesso em: 9 set. 2018.

\section{DADOS DOS AUTORES:}

\section{Cleudimar da Costa Silva}

Graduado em Fisioterapia. Instituto de Educação Superior Raimundo Sá (URSA). Picos/PI - Brasil. cleudimarcosta@hotmail.com 


\section{Virginia Leyla Santos Costa Urtiga}

Graduada em Enfermagem, Mestre em Farmacologia. Doutoranda em Farmacologia. Universidade Federal do Ceará (UFC). Fortaleza/CE - Brasil. iesrsa@faculdadersa.com.br

\section{André Pontes Silva}

Graduado em Educação Física. Mestrando em Saúde do Adulto. Universidade Federal do Maranhão. Maranhão/MA - Brasil. vozandrepontes@gmail.com

Submetido em: 2-5-2020

Aceito em: 26-9-2020 